PROCEEDINGS OF THE

AMERICAN MATHEMATICAL SOCIETY

Volume 133, Number 7, Pages 2005-2012

S 0002-9939(05)07870-6

Article electronically published on February 15, 2005

\title{
A NOTE ON EXPONENTIAL DECAY PROPERTIES OF GROUND STATES FOR QUASILINEAR ELLIPTIC EQUATIONS
}

\author{
YI LI AND CHUNSHAN ZHAO \\ (Communicated by David S. Tartakoff)
}

\begin{abstract}
We give an explicit formula for exponential decay properties of ground states for a class of quasilinear elliptic equations in the whole space $\mathbb{R}^{N}$
\end{abstract}

\section{INTRODUCTION}

We consider exponential decay properties of ground states of the quasilinear elliptic equation

$$
\Delta_{m} u+f(u)=0 \quad \text { in } \mathbb{R}^{N}, \quad N>m>1,
$$

where $\Delta_{m} u=\operatorname{div}\left(|D u|^{m-2} D u\right)$ is the degenerate $m$-Laplace operator. Here by a ground state we mean a non-negative non-trivial $C^{1}$ distribution solution of (1.1) which tends to zero at $\infty$.

Remark 1. There are some sufficient conditions which guarantee the existence of a positive radial ground state $u(x)$ satisfying $u(x)=u(r)$ with $r=|x|, u(0)=$ $\max _{x \in \mathbb{R}^{N}} u(x), u^{\prime}(0)=0$ and $u^{\prime}(r)<0$ for $r>0$, where $u^{\prime}(r)=\frac{d u(r)}{d r}$. See, for example, (H1) - (H3) in reference [6]. A good example for $f(u)$ which satisfies (A1) is:

$$
f(u)=-u^{m-1}+u^{q} \text { with } m-1<q<\frac{N(m-1)+m}{N-m} .
$$

From now on we make the following assumption on $f$ :

(A1): $f: \mathbb{R}^{+} \rightarrow \mathbb{R}$ is continuous and there exist positive constants $k$ and $\delta$ such that $f(z)+k z^{m-1}=O\left(z^{m-1+\delta}\right)$ as $z \downarrow 0$.

Before stating our results, let us recollect some facts about ground states for (1.1) with $m=2$, i.e.,

$$
\Delta u+f(u)=0 \text { in } \mathbb{R}^{N} .
$$

Received by the editors February 25, 2004.

2000 Mathematics Subject Classification. Primary 35B40, 35J70.

Key words and phrases. $m$-Laplace operator, ground states, exponential decay.

The first author was supported in part by the NSFC (10471052) and the Xiao-Xiang Funds of Hunan Normal University. 
Under suitable conditions on $f$ (e.g. [2]) it is well known that the ground state for (1.2) satisfies

$$
\begin{aligned}
& u(x)=u(|x|)=u(r)>0, u(0)=\max _{x \in \mathbb{R}^{N}} u(x), \\
& u^{\prime}(0)=0 \quad \text { and } \quad u^{\prime}(r)<0 \quad \text { for } \quad r \in(0, \infty)
\end{aligned}
$$

Moreover,

$$
\lim _{r \rightarrow \infty} u(r) r^{\frac{N-1}{2}} e^{r}=C_{1}
$$

where $0<C_{1}<\infty$ is a constant, and such precise estimates of asymptotes have been proved to be very useful. For example, for applications of such estimates for $m=2$, readers can refer to [1], 2], 3], [4, [5], etc. In this note we show that a similar estimate to (1.3) also holds for radial ground states of (1.1) if assumption (A1) is satisfied. Our main result is as follows.

Theorem 1.1. Let $u(x)=u(r)$ be a positive radial ground state for (1.1) and let $f(z)$ satisfy assumptions $(A 1)$. Then there exists a sequence of constants $\left\{c_{i}\right\}$ $(i=1,2, \cdots)$ such that

$$
\left(-\frac{u^{\prime}}{u}\right)^{m-1}=\varphi_{\infty}+\frac{c_{1}}{r}+\frac{c_{2}}{r^{2}}+\cdots+\frac{c_{l}}{r^{l}}+\cdots \quad \text { as } r \rightarrow \infty
$$

where $\left\{c_{i}\right\}(i=1,2, \cdots)$ are determined by

$$
c_{1}=\frac{N-1}{m}\left(\frac{k}{m-1}\right)^{\frac{m-2}{m}}, \quad c_{2}=\frac{(N-2) c_{1}-\frac{m}{2(m-1)} \varphi_{\infty}^{\frac{2-m}{m-1}} c_{1}^{2}}{m \varphi_{\infty}^{\frac{1}{m}}},
$$

and for $l>2, c_{l}$ can be uniquely determined by

$$
(N-l) c_{l-1}-m \varphi_{\infty}^{\frac{1}{m-1}} c_{l}=\sum_{i=2}^{l} \frac{F^{(i)}(0)}{i !}\left(\sum_{i_{1}+\cdots+i_{i}=l, i_{1}, \cdots i_{i}>0} c_{i_{1}} c_{i_{2}} \cdots c_{i_{i}}\right)
$$

where $\varphi_{\infty}=\left(\frac{k}{m-1}\right)^{\frac{m-1}{m}}$ and $F(\rho)=(m-1)\left(\varphi_{\infty}+\rho\right)^{\frac{m}{m-1}}$. In particular we have

$$
\lim _{r \rightarrow \infty} u(r) r^{\frac{N-1}{m(m-1)}} e^{\left(\frac{k}{m-1}\right)^{\frac{1}{m}} r}=C_{2}
$$

for some $0<C_{2}<\infty$.

\section{Proof of Theorem 1.1}

In this section, we will present the proof of Theorem 1.1. First let $u(x)=u(r)$ be a radial ground state as stated in Theorem 1.1. It follows from (1.1) that

$$
\left(\left|u^{\prime}\right|^{m-2} u^{\prime}\right)^{\prime}+\frac{N-1}{r}\left|u^{\prime}\right|^{m-2} u^{\prime}+f(u)=0 .
$$

Let $\varphi(r)=-\frac{\left|u^{\prime}\right|^{m-2} u^{\prime}}{u^{m-1}}$. Then $\left|u^{\prime}\right|^{m-2} u^{\prime}=-\varphi u^{m-1}$ and $\frac{u^{\prime}}{u}=-\varphi^{\frac{1}{m-1}}$. Substituting them into (2.1) yields

$$
\varphi^{\prime}-(m-1) \varphi^{\frac{m}{m-1}}+\frac{N-1}{r} \varphi-\frac{f(u)}{u^{m-1}}=0 .
$$

Lemma 2.1. $\lim \sup _{r \rightarrow \infty} \varphi(r)<\infty$. 
Proof. It follows from assumption (A1) and $\lim _{r \rightarrow \infty} u(r)=0$ that

$$
K=\sup _{r} \frac{|f(u(r))|}{u^{m-1}(r)}<\infty
$$

Noticing from $\varphi(r) \geq 0$ and (2.2) we obtain that as long as $\varphi(r) \geq\left(\frac{4 K}{m-1}\right)^{\frac{m-1}{m}}$ and $r \geq \frac{4(N-1)}{m-1}\left(\frac{m-1}{4 K}\right)^{\frac{m-1}{m}}$,

$$
\varphi^{\prime}=(m-1) \varphi^{\frac{m}{m-1}}-\frac{N-1}{r} \varphi+\frac{f(u)}{u^{m-1}} \geq \frac{m-1}{2} \varphi^{\frac{m}{m-1}} .
$$

Now, suppose to the contrary that $\lim _{\sup _{r \rightarrow \infty}} \varphi(r)=\infty$. Let

$$
r_{1}=\inf \left\{r \geq \frac{4(N-1)}{m-1}\left(\frac{m-1}{4 K}\right)^{\frac{m-1}{m}}: \varphi(r) \geq\left(\frac{4 K}{m-1}\right)^{\frac{m-1}{m}}\right\} .
$$

Since $\varphi(0)=0$ and $\varphi(r)>0$ for $r>0$, it follows that $0<r_{1} \leq \infty$. If $r_{1}=\infty$ we are done. Next we suppose $r_{1}<\infty$. Then $\varphi\left(r_{1}\right)=\left(\frac{4 K}{m-1}\right)^{\frac{m_{-1}}{m}}$ and $\varphi^{\prime}(r) \geq$ $\frac{m-1}{2} \varphi(r)^{\frac{m}{m-1}}$ for all $r \geq r_{1}$, which blows up before or at $r_{2}=r_{1}+2\left(\frac{m-1}{4 K}\right)^{\frac{1}{m}}$. This contradicts the well-definedness of $\varphi$. The proof of this lemma is completed.

\section{Lemma 2.2.}

$$
\lim _{r \rightarrow \infty} \varphi=\varphi_{\infty}=\left(\frac{k}{m-1}\right)^{\frac{m-1}{m}} .
$$

Proof. Since $\varphi(0)=0$ and $\varphi(r)>0$ for $r>0$ it follows from (2.21) and Lemma 2.1 that

and

$$
0 \leq \liminf _{r \rightarrow \infty} \varphi(r)=\alpha<\infty
$$

$$
0<\limsup _{r \rightarrow \infty} \varphi(r)=\beta<\infty .
$$

Next we use contradiction arguments to prove (2.4). Suppose

$$
\alpha=\liminf _{r \rightarrow \infty} \varphi(r)<\limsup _{r \rightarrow \infty} \varphi(r)=\beta .
$$

Then we may choose two sequences $\left\{\eta_{i}\right\}$ and $\left\{\zeta_{i}\right\}$ going to $\infty$ as $i \rightarrow \infty$ such that

$$
\left\{\eta_{i}\right\} \text { are local minima of } \varphi
$$

and

and

$$
\left\{\zeta_{i}\right\} \text { are local maxima of } \varphi
$$

$$
\eta_{i}<\zeta_{i}<\eta_{i+1} \text { and } \liminf _{r \rightarrow \infty} \varphi(r)=\lim _{i \rightarrow \infty} \varphi\left(\eta_{i}\right)=\alpha, \limsup _{r \rightarrow \infty} \varphi(r)=\lim _{i \rightarrow \infty} \varphi\left(\zeta_{i}\right)=\beta .
$$

Then at $\eta_{i}(i=1,2, \cdots)$ we know $u^{\prime}\left(\eta_{i}\right)=0$ and thus

$$
-(m-1) \varphi\left(\eta_{i}\right)^{\frac{m}{m-1}}+\frac{N-1}{\eta_{i}} \varphi\left(\eta_{i}\right)-\frac{f\left(u\left(\eta_{i}\right)\right)}{u\left(\eta_{i}\right)^{m-1}}=0 .
$$

Similarly at $\zeta_{i}(i=1,2, \cdots)$ we get

$$
-(m-1) \varphi\left(\zeta_{i}\right)^{\frac{m}{m-1}}+\frac{N-1}{\zeta_{i}} \varphi\left(\zeta_{i}\right)-\frac{f\left(u\left(\zeta_{i}\right)\right)}{u\left(\zeta_{i}\right)^{m-1}}=0,
$$


$\forall \epsilon>0$. Since $\lim _{z \downarrow 0} \frac{f(z)}{z^{m-1}}=-k$ and $\lim _{r \rightarrow \infty} u(r)=0$, we can take $r_{3}$ sufficiently large such that

Next we take $i_{0}$ sufficiently large such that

$$
\left|\frac{f(u(r))}{u(r)^{m-1}}+k\right|<\epsilon \quad \text { for all } r>r_{3} .
$$

$$
\eta_{i}>r_{3} \text { for } i>i_{0} \text {. }
$$

From (2.5) and (2.6) it follows that for all $i>i_{0}$ :

$$
k-\epsilon<(m-1) \varphi\left(\eta_{i}\right)^{\frac{m}{m-1}}-\frac{N-1}{\eta_{i}} \varphi\left(\eta_{i}\right)<k+\epsilon
$$

and

$$
k-\epsilon<(m-1) \varphi\left(\zeta_{i}\right)^{\frac{m}{m-1}}-\frac{N-1}{\zeta_{i}} \varphi\left(\zeta_{i}\right)<k+\epsilon .
$$

Letting $i \rightarrow \infty$ and noticing the arbitrariness of $\epsilon$, we get

$$
(m-1) \alpha^{\frac{m}{m-1}}=k, \quad(m-1) \beta^{\frac{m}{m-1}}=k,
$$

which yields $\alpha=\beta$, contradicting $\alpha<\beta$.

Therefore

$$
\lim _{r \rightarrow \infty} \varphi(r)=\varphi_{\infty}=\left(\frac{k}{m-1}\right)^{\frac{m-1}{m}} .
$$

The proof of this lemma is complete.

After presenting the above two lemmas we can now give a proof of our main result.

Proof of Theorem 1.1. We deduce from Lemma 2.2 that

$$
\lim _{r \rightarrow \infty} \frac{u^{\prime}}{u}=\lim _{r \rightarrow \infty}-\varphi^{\frac{1}{m-1}}=-\left(\frac{k}{m-1}\right)^{\frac{1}{m}} .
$$

Therefore $\forall \epsilon>0$, there exists a constant $0<c(\epsilon)<\infty$ such that

$$
u(r) \leq c(\epsilon) e^{-\left(\frac{k-\epsilon}{m-1}\right)^{\frac{1}{m}} r} .
$$

In particular taking $\epsilon=\frac{k}{2}$ we have

$$
u(r) \leq c(k) e^{-\left(\frac{k}{2(m-1)}\right)^{\frac{1}{m}} r} .
$$

For convenience, let $\left(\frac{k}{2(m-1)}\right)^{\frac{1}{m}}=\mu$. We know

$$
u(r) \leq c e^{-\mu r} .
$$

Next we give a more precise expansion of $\varphi(r)$ at $\infty$. Let $\varphi=\varphi_{\infty}+\varphi_{1}$. We know from (2.2) and Lemma 2.2 that $\lim _{r \rightarrow \infty} \varphi_{1}(r)=0$ and $\varphi_{1}(r)$ satisfies

$$
\varphi_{1}^{\prime}-(m-1)\left(\varphi_{\infty}+\varphi_{1}\right)^{\frac{m}{m-1}}+\frac{N-1}{r}\left(\varphi_{\infty}+\varphi_{1}\right)-\frac{f(u)}{u^{m-1}}=0,
$$

i.e.,

$$
\begin{gathered}
\varphi_{1}^{\prime}-m \varphi_{\infty}^{\frac{1}{m-1}} \varphi_{1}+\frac{N-1}{r} \varphi_{1} \\
=\frac{f(u)}{u^{m-1}}+(m-1)\left(\varphi_{\infty}+\varphi_{1}\right)^{\frac{m}{m-1}}-m \varphi_{\infty}^{\frac{1}{m-1}} \varphi_{1}-\frac{N-1}{r} \varphi_{\infty} .
\end{gathered}
$$


Noticing that $\lim _{r \rightarrow \infty} u(r)=0$ and $\frac{f(z)+k z^{m-1}}{z^{m-1}}=O\left(z^{\delta}\right)$ as $z \downarrow 0$, we get for $r$ sufficiently large that

$$
\frac{f(u)}{u^{m-1}}=-k+O\left(u^{\delta}\right)
$$

At the same time for $r$ sufficiently large we have

$$
(m-1)\left(\varphi_{\infty}+\varphi_{1}\right)^{\frac{m}{m-1}}=(m-1) \varphi_{\infty}^{\frac{m}{m-1}}+m \varphi_{\infty}^{\frac{1}{m-1}} \varphi_{1}+O\left(\varphi_{1}^{2}\right) .
$$

Thus from (2.8)-(2.10) it follows that for $r$ sufficiently large,

$$
\varphi_{1}^{\prime}-m \varphi_{\infty}^{\frac{1}{m-1}} \varphi_{1}+\frac{N-1}{r} \varphi_{1}=O\left(\varphi_{1}^{2}\right)-\frac{N-1}{r} \varphi_{\infty}+O\left(u^{\delta}\right) .
$$

Multiplying each side of (2.11) by $\varphi_{1}$ and integrating from $r$ to $\infty$ for $r$ sufficiently large, we get

$$
\begin{aligned}
& \frac{1}{2} \varphi_{1}^{2}(r)+\int_{r}^{\infty}\left(m \varphi_{\infty}^{\frac{1}{m-1}}-\frac{N-1}{s}+O\left(\varphi_{1}\right)\right) \varphi_{1}^{2} d s \\
& =\int_{r}^{\infty} \frac{N-1}{s} \varphi_{\infty} \varphi_{1} d s-\int_{r}^{\infty} O\left(u^{\delta}\right) \varphi_{1} d s .
\end{aligned}
$$

We can take $r$ sufficiently large such that

$$
m \varphi_{\infty}^{\frac{1}{m-1}}-\frac{N-1}{s}+O\left(\varphi_{1}\right) \geq \frac{1}{2} m \varphi_{\infty}^{\frac{1}{m-1}} \text { for } s>r .
$$

Therefore for such large $r$ it follows that

$$
\varphi_{1}^{2}(r)+\int_{r}^{\infty}\left(m \varphi_{\infty}^{\frac{1}{m-1}}\right) \varphi_{1}^{2} d s \leq 2 \int_{r}^{\infty} \frac{N-1}{s} \varphi_{\infty} \varphi_{1} d s-2 \int_{r}^{\infty} O\left(u^{\delta}\right) \varphi_{1} d s .
$$

Notice that

$$
2 \int_{r}^{\infty} \frac{N-1}{s} \varphi_{\infty} \varphi_{1} d s \leq \frac{1}{4}\left(m \varphi_{\infty}^{\frac{1}{m-1}}\right) \int_{r}^{\infty} \varphi_{1}^{2} d s+\frac{4(N-1)^{2} \varphi_{\infty}^{2}}{m \varphi_{\infty}^{\frac{1}{m-1}}} \int_{r}^{\infty} \frac{1}{s^{2}} d s
$$

and

$$
2 \int_{r}^{\infty} O\left(u^{\delta}\right) \varphi_{1} d s \leq \frac{1}{4}\left(m \varphi_{\infty}^{\frac{1}{m-1}}\right) \int_{r}^{\infty} \varphi_{1}^{2} d s+\frac{4 C}{m \varphi_{\infty}^{\frac{1}{m-1}}} \int_{r}^{\infty} O\left(u^{2 \delta}\right) d s .
$$

By virtue of the above estimates and (2.7) we obtain for $r$ sufficiently large,

$$
\begin{gathered}
\varphi_{1}^{2}(r)+\frac{1}{2}\left(m \varphi_{\infty}^{\frac{1}{m-1}}\right) \int_{r}^{\infty} \varphi_{1}^{2}(s) d s \\
\leq \frac{4(N-1)^{2} \varphi_{\infty}^{2}}{m \varphi_{\infty}^{\frac{1}{m-1}}} \frac{1}{r}+\frac{4 c}{2 \delta m \varphi_{\infty}^{\frac{1}{m-1}}} e^{-2 \delta \mu r} \\
\leq \frac{8(N-1)^{2} \varphi_{\infty}^{2}}{m \varphi_{\infty}^{\frac{1}{m-1}}} \frac{1}{r} .
\end{gathered}
$$

Thus we have

$$
\varphi_{1}^{2}(r)=O\left(\frac{1}{r}\right) \quad \text { as } r \rightarrow \infty .
$$

By this estimate and (2.11) it follows that as $r \rightarrow \infty$,

$$
\varphi_{1}^{\prime}-m \varphi_{\infty}^{\frac{1}{m-1}} \varphi_{1}+\frac{N-1}{r} \varphi_{1}=O\left(\frac{1}{r}\right) .
$$


For convenience let $\alpha_{0}=m \varphi_{\infty}^{\frac{1}{m-1}}$. Then we get from (2.15) as $r \rightarrow+\infty$,

$$
\left(r^{N-1} e^{-\alpha_{0} r} \varphi_{1}\right)^{\prime}=r^{N-1} e^{-\alpha_{0} r} O\left(\frac{1}{r}\right) .
$$

Integrating both sides of (2.16) from $r$ to $\infty$ yields as $r \rightarrow \infty$,

$$
\begin{aligned}
\varphi_{1}(r) & =\frac{e^{\alpha_{0} r}}{r^{N-1}} \int_{r}^{\infty} s^{N-1} O\left(\frac{1}{s}\right) e^{-\alpha_{0} s} d s \\
& =O\left(\frac{e^{\alpha_{0} r}}{r^{N-1}} \int_{r}^{\infty} s^{N-2} e^{-\alpha_{0} s} d s\right) .
\end{aligned}
$$

Applying integration by parts as many steps as we want we arrive that there exists a sequence of constants $\left\{a_{i}\right\}(i=1,2, \cdots)$ such that

$$
\begin{aligned}
\int_{r}^{\infty} s^{N-2} e^{-\alpha_{0} s} d s & =a_{1} r^{N-2} e^{-\alpha_{0} r}+a_{2} r^{N-3} e^{-\alpha_{0} r}+\cdots \\
& =e^{-\alpha_{0} r}\left(a_{1} r^{N-2}+a_{2} r^{N-3}+\cdots+a_{l} r^{N-l-1}+\cdots\right)
\end{aligned}
$$

where $a_{1}=\frac{1}{\alpha_{0}}$. Thus it follows from (2.16) that

$$
\varphi_{1}(r)=O\left(\frac{1}{r}\right) \text {, as } r \rightarrow \infty,
$$

which is an improvement of (2.14). Using (2.19) and (2.11) we obtain

$$
\left(r^{N-1} e^{-\alpha_{0} r} \varphi_{1}\right)^{\prime}=-r^{N-1} e^{-\alpha_{0} r}\left(\frac{N-1}{r} \varphi_{\infty}+O\left(\frac{1}{r^{2}}\right)\right)
$$

Similar to (2.16) and (2.17), we arrive at

$$
\begin{aligned}
\varphi_{1}(r) & =\frac{e^{\alpha_{0} r}}{r^{N-1}} \int_{r}^{\infty} s^{N-1} e^{-\alpha_{0} s}\left(\frac{N-1}{s} \varphi_{\infty}+O\left(\frac{1}{s^{2}}\right)\right) d s \\
& =\frac{(N-1) \varphi_{\infty} a_{1}}{r}+O\left(\frac{1}{r^{2}}\right) .
\end{aligned}
$$

Again if we let $\varphi_{1}=\frac{(N-1) \varphi_{\infty} a_{1}}{r}+\varphi_{2}$ such that $\varphi_{2}(r)=O\left(\frac{1}{r^{2}}\right)$, we obtain from (2.10) and (2.11) that

$$
\varphi_{2}^{\prime}-\alpha_{0} \varphi_{2}+\frac{N-1}{r} \varphi_{2}=\varphi_{\infty}^{\frac{m-2}{m-1}}\left[\frac{N-1}{2 m(m-1)}-\frac{N-2}{m}\right] \frac{N-1}{r^{2}}+O\left(\frac{1}{r^{2}}\right) .
$$

We can then repeat the same process to obtain the expansion as stated in Theorem 1.1 to any polynomial order as we want. Next we need to determine $c_{i}(i=1,2, \cdots)$ in Theorem 1.1 Let $F(\rho)=(m-1)\left(\varphi_{\infty}+\rho\right)^{\frac{m}{m-1}}$. Then the Taylor expansion of $F(\rho)$ at $\rho=0$ is as follows:

$$
\begin{gathered}
F(\rho)=(m-1) \varphi_{\infty}^{\frac{m}{m-1}}+\alpha_{0} \rho+\frac{m}{2(m-1)} \varphi_{\infty}^{-\frac{m-2}{m-1}} \rho^{2} \\
-\frac{m(m-2)}{3 !(m-1)^{2}} \varphi_{\infty}^{-\frac{2 m-3}{m-1}} \rho^{3}+\cdots+\frac{F^{(n)}(0)}{n !} \rho^{n}+\cdots
\end{gathered}
$$

where

$$
F^{(n)}(0)=\frac{(-1)^{n} m(m-2)(2 m-3) \cdots(l m-l-1) \cdots[(n-2) m-n+1]}{(m-1)^{n-1}}
$$


for $n \geq 4$. Thus from (2.8), (2.9) and (2.21) we get

$$
\begin{gathered}
\varphi_{1}^{\prime}-\alpha_{0} \varphi_{1}+\frac{N-1}{r} \varphi_{1}=O\left(u^{\delta}\right)-\frac{N-1}{r} \varphi_{\infty} \\
+\frac{m}{2(m-1)} \varphi_{\infty}^{-\frac{m-2}{m-1}} \varphi_{1}^{2}-\frac{m(m-2)}{3 !(m-1)^{2}} \varphi_{\infty}^{-\frac{2 m}{m-1}} \varphi_{1}^{3}+\cdots+\frac{F^{(n)}(0)}{n !} \varphi_{1}^{n}+\cdots
\end{gathered}
$$

Substituting

$$
\varphi_{1}(r)=\frac{c_{1}}{r}+\frac{c_{2}}{r^{2}}+\cdots+\frac{c_{l}}{r^{l}}+\cdots
$$

into (2.22) we get by comparing the coefficients of $\frac{1}{r^{n}}(n=1,2, \cdots)$ that

$$
c_{1}=\frac{N-1}{m}\left(\frac{k}{m-1}\right)^{\frac{m-2}{m}}
$$

and

$$
c_{2}=\frac{(N-2) c_{1}-\frac{m}{2(m-1)} \varphi_{\infty}^{-\frac{m-2}{m-1}} c_{1}^{2}}{\alpha_{0}} .
$$

$c_{l}(l>2)$ is determined by

$$
(N-l) c_{l-1}-\alpha_{0} c_{l}=\sum_{i=2}^{l} \frac{F^{(i)}(0)}{i !}\left(\sum_{i_{1}+i_{2}+\cdots+i_{i}=l} c_{i_{1}} c_{i_{2}} \cdots c_{i_{i}}\right) .
$$

Notice that

We know that as $r \rightarrow \infty$,

$$
\frac{u^{\prime}}{u}=-\varphi^{\frac{1}{m-1}}
$$

$$
\begin{aligned}
\frac{u^{\prime}}{u} & =-\left(\varphi_{\infty}^{\frac{1}{m-1}}+\frac{1}{m-1} \varphi_{\infty}^{\frac{2-m}{m-1}} c_{1} \cdot \frac{1}{r}+O\left(\frac{1}{r^{2}}\right)\right) \\
& =-\left(\frac{k}{m-1}\right)^{\frac{1}{m}}-\frac{N-1}{m(m-1)} \cdot \frac{1}{r}+O\left(\frac{1}{r^{2}}\right),
\end{aligned}
$$

which yields that

$$
u(r)=O\left(r^{-\frac{(N-1)}{m(m-1)}} e^{-\left(\frac{k}{m-1}\right)^{\frac{1}{m}} r}\right) \quad \text { as } r \rightarrow \infty,
$$

i.e.,

$$
\lim _{r \rightarrow \infty} u(r) r^{\frac{N-1}{m(m-1)}} e^{\left(\frac{k}{m-1}\right)^{\frac{1}{m}} r}=C_{2}
$$

for some $0<C_{2}<\infty$. The proof of Theorem 1.1 is complete.

\section{REFERENCES}

[1] S. Agmon, Lectures on exponential decay of solutions of second-order elliptic equations: bounds on eigenfunctions of $N$-body Schrödinger operators. Mathematical Notes, 29. Princeton University Press, Princeton, NJ; University of Tokyo Press, Tokyo, 1982. 118 pp. MR0745286 (85f:35019)

[2] B. Gidas, W. M. Ni, L. Nirenberg, Symmetry of positive solutions of nonlinear elliptic equations in $R^{n}$, Mathematical analysis and applications, Part A, pp. 369-402, Academic Press, New York-London, 1981. MF0634248 (84a:35083)

[3] Y. Li, Asymptotic behavior of positive solutions of equations $\Delta u+K(x) u^{p}=0$ in $R^{n}$, J. Differential Equations 95 (1992), No. 2, pp. 304-330. MF1165425 (93k:35048)

[4] W. M. Ni, I. Takagi, On the shape of least energy solutions to a semi-linear Neumann problem, Comm. Pure Appl. Math 44 (1991), No. 7, 819-851. MR1115095(92i:35052) 
[5] W. M. Ni, J. Wei, On the location and profile of spike-layer solutions to singularly perturbed semi-linear Dirichlet problems, Comm. Pure Appl. Math. 48 (1995), No. 7, 731-768. MR1342381 (96g:35077)

[6] J. Serrin, M. Tang, Uniqueness of ground states for quasilinear elliptic equations, Indiana Univ. Math. J. 49 (2000) No. 3, pp. 897-923. MR1803216 (2002d:35072)

Department of Mathematics, Hunan Normal University, Changsha, People's Republic of China - and - Department of Mathematics, The University of Iowa, Iowa City, Iowa 52242

E-mail address: yli@math.uiowa.edu

Department of Mathematics, The University of Iowa, Iowa City, Iowa 52242

E-mail address: chuzhao@math.uiowa.edu 